\title{
RAC 1: Análisis de un caso de éxito de emisora de radio
}

\section{$R A C$ 1: keys to the success of a radio station}

\author{
Sílvia Espinosa-Mirabet; Maria Gutiérrez; Josep-Maria Martí-Martí
}

Cómo citar este artículo:

Espinosa-Mirabet, Sílvia; Gutiérrez, Maria; Martí-Martí, Josep-Maria (2021). “RAC 1: Análisis de un caso de éxito de emisora de radio". Profesional de la información, v. 30, n. 3, e300303.

https://doi.org/10.3145/epi.2021.may.03

Artículo recibido el $17-07-2020$

Aceptación definitiva: 19-09-2020

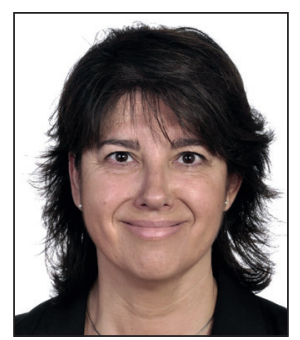

Sílvia Espinosa-Mirabet

https://orcid.org/0000-0002-8304-5882

Universitat de Girona

Dept. de Filologia i Comunicació

Membre SGR 2017-00385 Targetslab-GREP

Campus del Barri Vell, Edif. St. Domènec

PI. Ferreter Mora, 1. 17004 Girona, España

silvia.espinosam@udg.edu

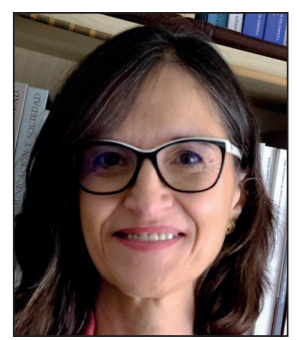

Maria Gutiérrez

https://orcid.org/0000-0002-2340-7677

Universitat Autònoma de Barcelona (UAB)

Departamento de Comunicación

Audiovisual y Publicidad

Edif. I, carrer de la Vinya

08193 Bellaterra (Barcelona), España

maria.gutierrez@uab.cat

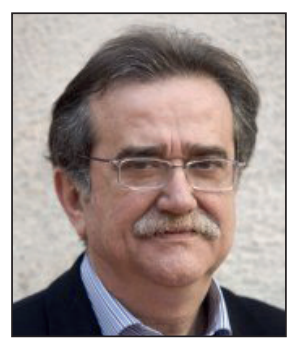

Josep-Maria Martí-Martí

https://orcid.org/0000-0003-2533-5109

Universitat Autònoma de Barcelona (UAB)

Departamento de Comunicación

Audiovisual y Publicidad

Edif. I, carrer de la Vinya

08193 Bellaterra (Barcelona), España

josepmaria.marti@uab.cat

\section{Resumen}

Se identifican y explican los factores que han contribuido a posicionar a RAC 1, emisora del Grupo Godó, como líder de audiencia en Cataluña a pesar de ser la última cadena incorporada al dial. El análisis pormenorizado de los datos de audiencia de la radiodifusión en catalán es una línea de investigación del Observatori de la Ràdio a Catalunya. En los últimos 10 años se ha observado un aumento de los oyentes que escuchan radio solo en catalán, con independencia de su lengua de comunicación habitual. Este fenómeno es inédito y requiere ser analizado más allá de interpretaciones casuales. Según datos del Estudio General de Medios (EGM), RAC 1 ocupa más de un $40 \%$ del share diario de la radio generalista (2019), situándose como primera preferencia para los oyentes en Cataluña. Además, se ha convertido en la 5 a emisora de radio convencional (837.000 oyentes) en España, con muchos más oyentes de los que tienen cadenas de cobertura estatal. RAC 1 cumplió 20 años el 1 de mayo de 2020. Enfoca sus contenidos en base al infoentretenimiento y tiene una imagen de éxito entre los medios de comunicación. Abordar y describir las claves del éxito de una emisora de radio es un enfoque nuevo en la bibliografía académica. Hacerlo a partir del estudio de caso de RAC 1, que lleva una década ininterrumpida siendo líder de audiencia, es la primera vez que se hace. Para conocer las causas de este fenómeno se ha utilizado una metodología que analiza el posicionamiento de la cadena en el conjunto de la oferta, sus estrategias de programación y, por último, sus contenidos programáticos, en base a los informes del Observatori de la Ràdio a Catalunya, del Institut de la Comunicació (InCOM) de la Universitat Autònoma de Barcelona (UAB) y de datos de la Generalitat de Catalunya. El análisis se ha completado con entrevistas en profundidad a los principales realizadores.

\section{Palabras clave}

RAC1; RAC 1; Audiencia; Liderazgo radiofónico; Infoentretenimiento; Radio; Radio en catalán; Nuevos formatos radiofónicos; Radio digital; Radio analógica; Contenidos creativos; Claves de éxito; Factores de éxito; Estrategias; Cataluña.

\section{Abstract}

The factors that have contributed to the position of RAC 1, a radio station belonging to the Godó Group, as the audience leader in Catalonia, despite being the most recent channel available, are defined and explained. This detailed analysis of audience data for Catalan broadcasting is a line of research carried out by the Observatori de la Ràdio a Catalunya. The last 10 years have seen an increase in the number of people who listen to radio only in Catalan, regardless of the 
language they typically use for communication. This phenomenon is unprecedented and requires an analysis beyond casual interpretations. According to EGM data, RAC 1 accounts for more than $40 \%$ of the daily share of talk radio (2019), placing it as the first preference for listeners in Catalonia. In addition, this radio station has become the fifth most important conventional radio station $(837,000$ listeners) in Spain, with many more listeners than stations that have national coverage. RAC 1 celebrated its 20th birthday on 1 May 2020. Its contents focuses on infotainment and has a successful image among the media. Addressing and describing the keys to the success of a radio station represents a new approach in the scholarly literature. This case study of $R A C 1$, which has been an audience leader for a decade, thus represents a pioneering approach. The methodology applied to determine the causes of this phenomenon includes an analysis of the positioning of the channel's overall offering, its programming strategies, and its program content, based on reports from the Observatori de la Ràdio a Catalunya, the Institut de la Comunicació (InCOM) of the Universitat Autònoma de Barcelona (UAB), and the Generalitat de Catalunya. The analysis is completed by in-depth interviews with the main stakeholders of the radio station.

\section{Keywords}

RAC1; RAC 1; Audience; Radio leadership; Infotainment; Radio stations; Radio in Catalan; New radio formats; Digital radio; Analog radio; Creative radio content; Success factors; Keys to success; Strategies; Catalonia; Catalunya.

\section{Financiación}

Proyecto financiado por el Observatori de la Ràdio a Catalunya.

\section{Introducción y contexto teórico ${ }^{1}$}

El 1 de mayo de 2020, RAC 1 (siglas correspondientes a Ràdio Associació de Catalunya) cumplió 20 años y lo celebró en antena, a pesar del confinamiento de parte de sus presentadores estrella y de su plantilla de más de 100 personas. Esta emisora generalista y privada, con emisión $100 \%$ en catalán, aparte de ser líder de audiencia, ha revolucionado la manera de hacer radio y no sólo en Cataluña. De entrada, lo conseguido parece fuera de dudas: un liderazgo de audiencia ininterrumpido durante 11 años, sumando oyentes catalano y castellanoparlantes, nuevas estrategias de relación con sus oyentes (comunidad) y, por último, la consecución reconocible de un tono especial de antena, el cual ha provocado, en cierto modo, que su competencia directa, Catalunya Ràdio, tuviera que actualizar sus estructuras programáticas hasta aquel momento inamovibles. El programa informativo matinal, El matí de Catalunya Ràdio es un buen ejemplo (Gutiérrez, 2014, p. 84). Después de la marcha del periodista Antoni Bassas (sustituido por Neus Bonet) y de la debacle de audiencia por la competencia de $R A C$ 1, la emisora de la Generalitat apostó por Manel Fuentes que le dio un toque mucho más distendido al espacio, más en la línea imperante en $R A C 1^{2}$.

El análisis de diferencias no se puede hacer sólo a partir de datos de audiencia. Las cifras sobre el consumo de radio arrojan resultados de distinta índole que, en la práctica profesional se convierten en variables interpretables según los intereses de cada operador. En realidad, la mayoría de los datos por sí mismos aportan escasa información para poder entender las razones por las cuales una marca aumenta o reduce su share. Pese a todo lo antedicho, la publicación de esta información continúa siendo crucial para las empresas radiofónicas porque, aun encontrándose su modelo programático en un proceso de transformación, su posicionamiento en el mercado analógico sigue siendo fundamental para mantener sus ingresos publicitarios, mientras se plantean las estrategias de futuro (Fradin, 2016; Martí-Martí et al., 2016). Todavía la radio más escuchada sigue siendo la emitida por frecuencia modulada.

"La escucha de la radio por otros canales de distribución más allá de la frecuencia modulada es aún minoritaria, pero ya significativa, aunque se combina con la escucha tradicional. Por primera vez, en 2018 el EGM (Estudio General de medios) recoge datos sobre consumo de radio por streaming y en formato podcast" (Llorens-Maluquer, 2019, p. 173).

De hecho, IAB Spain (2019) constata que el universo hertziano se extiende hacia el entorno online, en el que "destacan las cadenas de radio convencional de mayor audiencia". Partiendo de dicha premisa, ¿qué factores provocan que los oyentes tiendan a consumir una u otra oferta?

No existen publicaciones científicas que respondan a este interrogante de forma taxativa y pormenorizada, aunque sí que se han identificado algunos trabajos (Gascón-Vera, 2016) que enumeran las características que se le presuponen a un programa televisivo para tener posibilidades de éxito. Algunas de estas claves pueden extrapolarse al entorno radiofónico. Así, Martí-Martí (2016) entiende que los aspectos que contribuyen al éxito en un programa de radio son, entre otros: la adecuada selección del target de audiencia al que se dirige, la buena elección de un equipo de producción y los aspectos diferenciales:

"determinar cuáles van a ser sus elementos originales, es decir, aquellos que van a interesar a los oyentes y lo diferenciarán de la competencia" (Martí-Martí, 2016, pp. 48-49).

Otro aspecto clave es la calidad (Pujadas, en Gascón, 2016), una característica que engloba items tan esenciales como la innovación, la experimentación, el no repetir fórmulas e incluso el ofrecer productos fascinantes y profundos. Videla y Sanjuán (2006) añaden a la lista el humor, aspecto que comparte Durán (2015) cuando define show radiofónico a partir de José-Luis Fernández: 
"una escena poblada por humoristas, locutores, especialistas en distintos temas..." coordinados todos por "... el conductor que se destaca en este contexto, más por su carácter de coordinador y emblema del programa, que por un saber específico que lo distinga. En este espacio de intensa sociabilidad afectiva y laboral, es en el marco en que se van a producir la aparición de múltiples géneros breves, algunos radiofónicos y otros que tienen vida propia: noticias, comentarios, chistes, sketches humorísticos, canciones, tandas publicitarias, recetas culinarias, etc. (...) vemos el show radiofónico como un gran género en el cual se combinan otros géneros o fragmentos de ellos" (Durán, 2015 p. 3).

El éxito de muchos de estos espacios radiofónicos que, desde el humor, analizan la actualidad radica en la creación de un vínculo emocional con sus oyentes que genera

"un acuerdo de complicidad a fin de descalificar y cuestionar los valores de los poderosos. Con ello, la irritación y molestias sociales son encauzadas" (Medina, 2017, p. 177).

Desde esta perspectiva, la fidelización de la audiencia implica además y, desde los albores de la radio (Espinosa-Mirabet, 2013) poder disponer de una línea comercial, fácilmente explotable. Asimismo, el entretenimiento como género principal, concede algunas ventajas competitivas además de que el programa consiga la máxima identificación con su audiencia regular (Toledo en Guerrero-Pérez, 2013). Según Basté (2011), RAC 1 da una vuelta al entretenimiento y nace con una programación basada en gran parte en el infoentretenimiento, género híbrido que conjuga la actualidad con contenidos asociados al entretenimiento y que tiene su máximo exponente en el magazín. (Huertas; Gutiérrez, 2003, p. 122).

Por su parte Martí-Martí (2000) destaca la conveniencia de conseguir una personalidad global de realización. Es decir, diseñar un carácter sonoro propio a partir de parámetros como el estilo de expresión y de locución (tono, recursos narrativos, participación de los presentadores) y los logos sonoros. Acorde a todo lo expuesto, las características que pueden contribuir al éxito de un producto audiovisual son:

- calidad, concepto que integra originalidad, innovación y una buena factura radiofónica (realización, sonido, etc.);

- inclusión en el relato de elementos de entretenimiento;

- proximidad con la audiencia en el sentido de no sólo de referenciarla, sino también de apelarla;

- humor;

- sencillez;

- preparación (guion e ideas);

- un presentador o presentadores que sepan conducir la mezcla; y

- que el formato haga su aparición en el mercado de la oferta y de la audiencia en el momento adecuado, es decir, que sea oportuno (Martí-Martí, 2000).

El objetivo de este artículo es averiguar por qué $R A C 1$ es líder de audiencia, a partir de profundizar en la interrelación programación radiofónica y audiencia. Su liderazgo ${ }^{3}$ hay que inscribirlo en el marco de otro hecho relevante: el ascenso progresivo del consumo de radio en catalán, cuya penetración pasó del 40,6\% en 2006 al 64\% en 2019 según datos del Estudio General de Medios (EGM)-Cataluña, referidos a las tres cadenas generalistas que sólo emiten en catalán (RAC 1, Catalunya Ràdio y $R 4$ de Radio Nacional de España, RNE). Del 64\% de penetración actual de la radio en catalán, y según cifras de AIMC-EGM, sólo RAC 1 acumula el 41,3\%.

\section{El entorno de RAC 1: los operadores en catalán en un entorno sociolingüístico diglósico}

El mercado de radio en Cataluña es un mercado maduro en términos de oferta (Martí-Martí, 2004). Esto significa que es complejo en su oferta programática, muy competitivo por el número de operadores en presencia (públicos, privados y tercer sector) y por el volumen de negocio que genera (Martí-Martí; Monclús, 2016). Además, supone el 20 \% de la audiencia total de la radio española y un $11 \%$ de su mercado publicitario en plena tendencia optimista ya que

"de los 48 millones de euros en 2016 ha pasado a los 50,7 millones de 2018, lo que supone un incremento del 4,9\%, aunque el retroceso de 2018 respecto de 2017 impidió que el aumento fuera mayor (ACR, 2019 en Llorens-Maluquer, 2019, p. 166).

Según los datos del Boletín de información sobre el audiovisual en Catalunya (BIAC, 2019) que depende del Consell de I'Audiovisual de Catalunya (CAC), un hogar catalán puede alcanzar a escuchar más de 30 emisoras de radio legales (y otras tanto piratas o ilegales). Ello es posible por la compleja estructura del mercado radiofónico compuesto por 262 emisoras de radio, de las cuales 12 tienen cobertura autonómica, 19 forman parte de cadenas estatales y del resto de las estaciones operativas, 231 son públicas y locales, y 16 locales y privadas (BIAC, 2019). Desde la óptica de la lengua vehicular y cobertura autonómica, emiten el 100\% de la programación en catalán las dos emisoras públicas con programación generalista [Ràdio 4 del Grupo RNE (Radio Nacional de España) y Catalunya Ràdio de la CCMA (Corporació Catalana de Mitjans Audiovisuals), dependiente de la Generalitat de Catalunya] y la privada RAC 1. Además, existen tres estaciones privadas, de grupos mediáticos con cabecera principal en Madrid que emiten programación bilingüe, en catalán y en español en función de una segmentación horaria que les permite cumplir con la normativa autonómica de producir en catalán 9 horas de su programa-

RAC 1 con 20 años de historia lleva más de una década siendo líder absoluto de audiencia en Catalunya 
ción diaria. Son: SER Catalunya de la Cadena SER, Onda Cero Cataluña, de la Cadena Onda Cero -Grupo Atresmedia y COPE Cataluña, de la Cadena COPE.

RAC 1 pertenece al Grupo Godó y es la emisora catalana más joven. Tiene una única sede en Barcelona y no cuenta con delegaciones territoriales. Entró en el mercado radiofónico español el 1 de mayo de 2000. Dos años más tarde, el EGM le otorgó una audiencia de 70.000 oyentes, que incrementó significativamente en la temporada 2010, al alcanzar los 510.000 y superando entonces la cifra de Catalunya Ràdio, hasta aquel momento la radio líder en Cataluña. Esta situación se ha mantenido a lo largo de los diez años siguientes y, a juzgar por los datos de la tercera ola del EGM, ha consolidado su primer puesto del ranking en 2019: RAC 1 suma 834.00 oyentes, Catalunya Ràdio 563.000 oyentes y la Cadena SER 343.000 (RAC, 2019).

Un aspecto importante a tener en cuenta para entender el panorama radiofónico catalán es el contexto sociolingüístico en Cataluña. A principios del año 2000, el uso social del catalán y del español estaba más o menos equilibrado, mientras que una década más tarde, en 2013, se comenzó a observar un retroceso en el uso de la lengua catalana (41\%) frente al de español (51\%). Según Pradilla y Sorolla $\left(2016^{4}\right)$, este cambio obedecía a tres factores demográficos:

1) el incremento de población originaria de otros lugares, que mayoritariamente se integraron utilizando básicamente la lengua española;

2) la baja tasa de natalidad de las familias autóctonas que alcanzaban porcentajes por debajo de la media europea; y

3) la aparición de nuevas prácticas sociales por parte de sectores como los jóvenes catalanoparlantes que han venido usando formas bilingües para expresarse de forma habitual y coloquial.

En referencia a este último factor, el Institut d'Estadística de Catalunya (Idescat, 2019), indica que únicamente el 36,10\% de la población mayor de 15 años, tiene el catalán como lengua habitual, aunque el 94,4\% lo entiende y el 81,2\% manifiesta hablarlo.

Quizá sea por ese nivel de comprensión que la lengua catalana ocupe un lugar tan relevante en las preferencias radiofónicas de la audiencia en Cataluña. De una manera no sistematizada, la industria radiofónica se ha encargado por su cuenta de crear los soportes técnico-lingüísticos para ayudar a periodistas, guionistas, etc., a desarrollar su labor y a generar un vocabulario y nuevas expresiones que describieran un mundo que hasta el momento solo había sido contado en español (CCMA, 2013, p. 7). Y es que tanto Ràdio 4 (RNE) como Catalunya Ràdio (CCMA), en los albores de la democracia, contribuyeron también a la recuperación de la lengua y cultura catalana (Corominas et al., 2005), un camino iniciado en 1979 por Ràdio Arenys, la primera emisora municipal. Así, puede afirmarse que la puesta en marcha de los medios públicos en catalán, no solo ha generado un estándar lingüístico propio, sino que además ha incrementado la recepción radiofónica en catalán (Espinosa-Mirabet; Gutiérrez; Martí-Martí, 2019).

\section{El éxito obtenido se debe según sus rea- lizadores estrella a su cercanía con el oyente, pero también a la renovación de los contenidos y de los formatos de pro- gramas de la radio generalista}

\section{Objetivos y metodología}

$R A C$ 1, la emisora generalista más joven del mercado radiofónico catalán, lleva una década (la mitad de su vida) ocupando el primer lugar en el ranking de audiencia. Este texto se plantea como objetivo identificar los factores que han influido en su liderazgo y que, acorde con los datos, lo han consolidado. Este estudio de caso podría servir para facilitar algunas claves que permitieran la renovación narrativa del sector de la radio convencional que no se caracteriza precisamente por su alto nivel de innovación, ni por su engagement con el target juvenil, aspecto que preocupa mucho a la Asociación Española de Radiodifusión Comercial (AERC) y a la Associació Catalana de Ràdio (ACR), por poner sólo dos ejemplos.

Para analizar las claves del éxito de $R A C 1$, se plantearon las siguientes preguntas de investigación:

RQ1: En tanto que emisora de radio convencional, ¿̇los contenidos de RAC 1 siguen los mismos parámetros que el resto de sus competidoras?

RQ2: ¿El uso del catalán como lengua vehicular es un factor determinante para entender su éxito?

RQ3: El nacimiento de RAC 1 coincide con la eclosión del mundo de internet, ¿̇hasta qué punto este hecho ha favorecido su éxito entre la audiencia?

Para poder dar respuesta a estas preguntas, se ha creado un instrumento metodológico que ha permitido un abordaje cuantitativo-cualitativo, identificando los parámetros que definen la oferta de contenidos de RAC 1 para entender cuáles son las claves de su éxito. Ello ha sido posible a partir de la revisión de los estudios centrados en el análisis de la oferta, elaborados tanto por el Consell de l'Audiovisual de Catalunya (CAC, 2001-2019) como por l'Observatori de la Ràdio (OBS, 2006-2014). Además, se han revisado también los informes elaborados desde el Observatori de la Llengua por Pradilla y Sorolla (2016) en relación al posicionamiento, conocimiento y reconocimiento lingüístico de la lengua catalana entre la potencial audiencia de Cataluña y los apartados de radio de los informes de la comunicación en Cataluña del Institut de la Comunicació (InCOM) de la Universitat Autònoma de Barcelona (UAB) (2009-2010 a 2017-2018). Finalmente, se han realizado entrevistas semiestructuradas a los directores de programas de $R A C 1$, lo que ha permitido confrontar sus 
comentarios con los datos obtenidos de la revisión bibliográfica. Todo ello para examinar los siguientes aspectos, que en un plano teórico (Martí-Martí, 2016; Gascón, 2016) se atribuyen al éxito de un programa audiovisual:

- Calidad de la oferta programática atendiendo a criterios de innovación radiofónica, originalidad y de no repetición de fórmulas conocidas.

- Presencia de los géneros principales, información y entretenimiento y su hibridación.

- Creación de comunidad. Cómo se teje la relación con los oyentes y a partir de qué recursos.

\section{Los aspectos singulares de RAC 1}

\subsection{Estructura de su programación}

En todos los análisis de programación revisados, RAC 1 es considerada como una emisora generalista que se dirige como tal a una audiencia heterogénea (Martí-Martí, 2004). Algunos de los adjetivos que popularmente se le atribuyen a RAC 1 para justificar su liderazgo es que suena a radio joven, fresca y más dinámica o entretenida que el resto de las emisoras generalistas convenciona-

RAC 1 ha contribuido al aumento del consumo de radio en catalán, ya que esta cadena aporta el $41,3 \%$ sobre el $64 \%$ total de penetración de la radio en esta lengua

les. Y estos epítetos fueron fundacionales, tal como recogía el diario La vanguardia en motivo del 20ㅇ aniversario de la emisora.

"La filosofía es que, después de escuchar RAC 1, el oyente se sienta bien. Esta es la atmósfera que se tenía que crear" (Morros, 2020).

Esta percepción se puede validar escuchando su programación, codificando algunos elementos identificadores y comparándolos con los de sus competidoras en el territorio (Gutiérrez, 2016, p. 67). Pero desde la mirada del periodista Albert Om, realizador del magazine de tarde Islàndia y uno de los pioneros de la emisora, RAC 1

"es una radio que ha apostado siempre de forma arriesgada por una oferta distinta. Por ejemplo, cuando yo empecé en 2000, en mi programa matinal L'hora del pati no había tertulias, y era una cosa muy diferente en aquel momento. Marcamos un tono, que se ha sabido mantener a lo largo de los años" (A. Om, entrevista personal, 25 de abril de 2020).

Esta forma de 'hacer radio diferente' les llevó, por ejemplo, a apostar por una creativa y ahorradora manera de tener programación 24h, pero a bajo coste. En la temporada 2005, el resto de emisoras que se oían en Cataluña comenzaban a reforzar la redifusión de sus principales programas como estrategia de parrilla, pero en RAC 1 ya se estilaba hacía tiempo, puesto que casi una cuarta parte de la oferta que se programaba entre la madrugada y el fin de semana eran programas ya emitidos. Los géneros que se incluían en reemisión eran: información, entretenimiento, deporte y humor (CAC, 2004, p. 119). En este sentido, hay que subrayar que RAC 1 continuó apostando por el género humor cuando para el resto de emisoras generalistas su presencia era testimonial o nula (Gutiérrez, 2008). El humor como ingrediente principal de varios programas de la estación es un rasgo definidor de la cadena desde sus comienzos y es indisociable de los gustos programáticos de los oyentes jóvenes universitarios que tiene RAC 1 (Pellicer-Muñoz, 2012).

También la interrelación franjas horarias y contenidos seguía su propia lógica en $R A C 1$, por lo que un programa podía comenzar su emisión en una franja horaria y acabarla en otra. Un ejemplo es el deportivo diario Primer toc, que hasta la temporada 2016 , entraba en antena a las 19:00 y finalizaba a las 21:00 horas, cuando tradicionalmente son las 20:00 horas el punto horario que marca el final de la tarde y el inicio de la programación de la noche. Esta estrategia le permitía diferenciarse de la oferta del resto de emisoras/cadenas. En 2016 fue sustituido por Islàndia, un magazín de infoentretenimiento que adoptó ese mismo horario, pero acortándolo 30 minutos e integrando en su inicio un bloque informativo de actualidad para desbancar al boletín horario. Esta fue una apuesta arriesgada ya que, por una parte, se eliminaba el tradicional deportivo vespertino y, por otra, se estrenaba un programa de 90 minutos, algo bastante insólito en la programación de lunes a viernes de las emisoras generalistas. Es cierto que otras emisoras/cadenas han presentado también cambios estructurales, en especial en la franja tarde, pero mayoritariamente se han dedicado a ampliar la duración del magazine correspondiente, de manera que quedase integrado como contenido propio el programa deportivo que proseguía al informativo del mediodía, entre otras (Gutiérrez, 2016, p. 72).

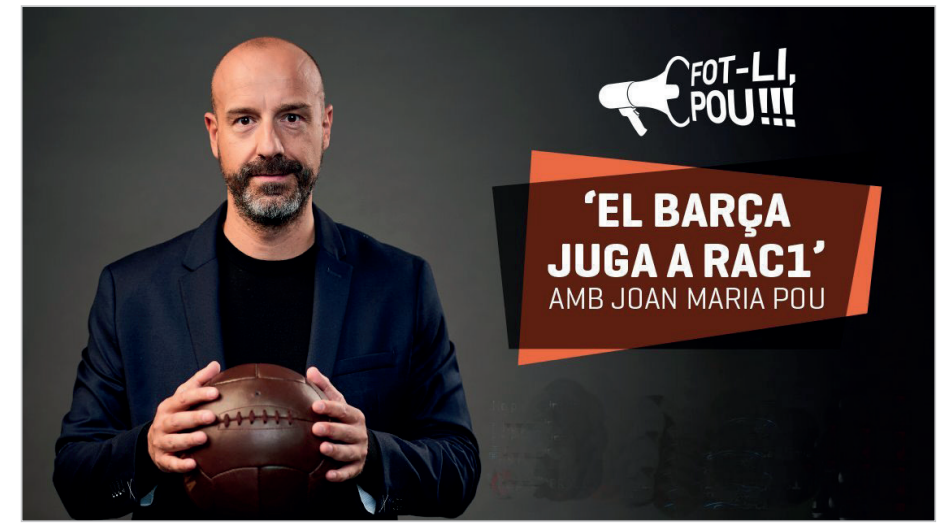

https://www.facebook.com/FCBRAC1 


\subsubsection{No siempre 'suenan los pitos'}

En $R A C$ 1, los boletines informativos no necesariamente coinciden con la hora en punto, emblema clásico de la radio generalista. Según el periodista de deportes y encargado de las retransmisiones del Barça, Joan-Maria Pou (2020),

"existen franjas en la programación que, si por contenido hace falta, se saltan... igual que los pitos. Y la gente lo ha integrado" (Joan M. Pou, entrevista personal, 10 de marzo de 2020).

De alguna manera, la narrativa del continuum está por encima de franjas programáticas aplicando un cierto sentido de flexibilidad en el momento de la emisión. Volviendo a los boletines horarios, estos pueden alterarse porque el contenido del programa, todavía en antena, no ha finalizado o porque minutos antes del punto horario se inició una entrevista de inminente actualidad. Esta práctica es una tendencia habitual en el magazine de prime time El món a RAC 1. Se trata de una dinámica introducida por RAC 1, que al cabo de un tiempo ha sido adoptada puntualmente en otras emisoras convencionales, que incluso han llegado a ofrecer también un

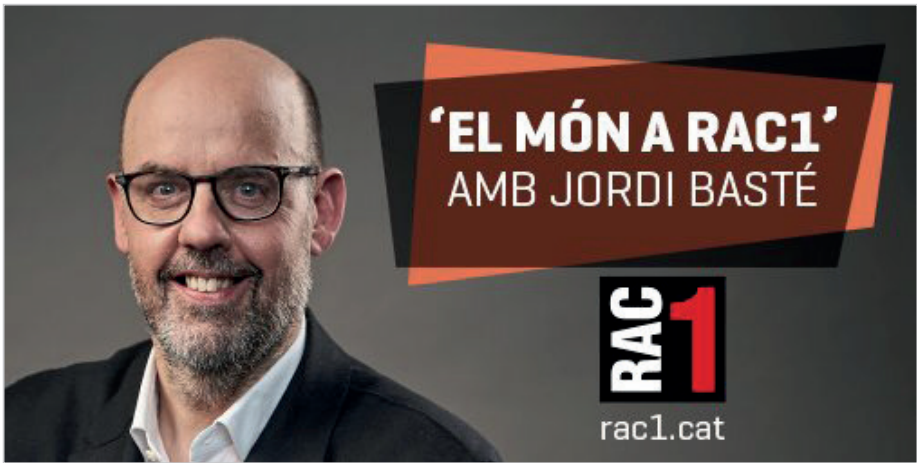

https://www.rac1.cat/programes/el-mon/20201102/49161166115/davantaljordi-baste.html

punto informativo a la media hora como, por ejemplo, era el caso de La tarde con Ramón García (COPE) en la temporada 2013 (Gutiérrez, 2016, p. 72).

\subsection{Peso de los géneros}

Como emisora generalista, RAC 1 fundamenta su parrilla de programación en los tres géneros básicos (Gutiérrez, 2016), Información, Deporte e Infoentretenimiento, al igual que lo hace el resto de emisoras/cadenas. La tipología de géneros se corresponde a la propuesta por Huertas y Gutiérrez (2003).

\subsubsection{Infoentretenimiento para acompañar}

Desde una perspectiva histórica, $R A C 1$ ha tendido a buscar un cierto equilibrio entre los tres géneros básicos, y una consecuencia de ello ha sido la reducción de programas informativos. Otra, la presencia de la opinión de los realizadores en los programas de información como reconoce el director de El món a RAC 1, Basté (2011). Sus editoriales de las 8:00h, EI davantal, son argumentos que tiñen buena parte de la programación de la emisora, pero también de la agenda setting del día. La opinión de Basté durante diferentes espacios de la emisión de su programa (en las tertulias, por ejemplo) es un rasgo característico.

En términos más rígidos, el género Información en la temporada 2019-2020 articula el informativo 14/15, con una duración de 15 minutos, y el de noche No ho sé, de 120 minutos, una circunstancia que es heredada de temporadas anteriores. El bajo volumen de presencia de contenidos informativos regidos desde un título de programas difícilmente se encuentra, no sólo en Catalunya Ràdio, sino también en las cadenas SER, COPE y Onda Cero. A modo de ejemplo, el gráfico 1 muestra una comparativa entre la oferta de RAC 1 y Catalunya Ràdio. En la emisora pública, la Información prevalece por encima del género Deporte y del híbrido Infoentretenimiento, mientras que en $R A C$ 1 se observa lo contrario. Este último planteamiento se asemeja bastante a la del resto de las cadenas que operan en el ámbito estatal (Gutiérrez, 2016). Sin embargo, ha parecido interesante comparar las parrillas de la primera y segunda emisoras en el ranking de Cataluña en una único gráfico para tener una perspectiva histórica de los datos (gráfico 1).

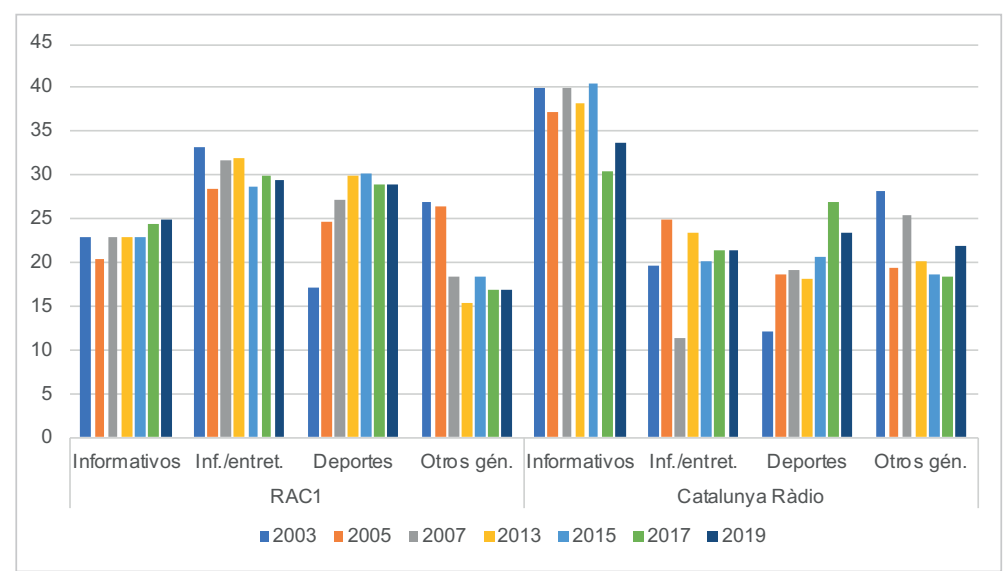

Gráfico 1. Oferta según el género.

Fuente: Elaborado a partir de Informes CAC, Observatori de la Ràdio a Catalunya y análisis de la oferta de RAC 1 y Catalunya Ràdio.

\subsubsection{Creciendo y evolucionando}

A la vista de los resultados obtenidos, la pregunta es ¿cómo consigue RAC 1 mantener una propuesta más equilibrada que el resto de emisoras/cadenas en relación a los géneros básicos? El peso de la Información como género ha sido uno de los aspectos que más han variado en RAC 1 desde su creación. Al principio la programación giraba en torno a Deportes y Entretenimiento, 
"pero existe un momento en el cual se da un salto hacia una radio de referencia informativa, pero se mantiene el tono (se refiere al tono distendido de la programación)... se cuida más allá del entretenimiento, se mira la actualidad y el análisis. Y en ese momento se crece por todos lados (J. M. Pou, entrevista personal, 10 de marzo de 2020).

En palabras del director del programa líder de tardes, Versió RAC1, Toni Clapés, la programación de RAC 1

"es como una especie de radiofórmula de presentadores que entretienen e informan" (T. Clapés, entrevista personal, 10 de mayo de 2020).

Para él, la información en RAC 1 es muy puntual y casa con los gustos de su público.

"Cuando la gente quiere información, cuando buscan información pura y dura, ya hay otras referencias, o a través de la Red u otros canales" (T. Clapés, entrevista personal, 10 de mayo de 2020).

La información es más protagonista en el espacio matinal de Jordi Basté. En este sentido, cuando RAC 1 cambió su web en 2016 para convertirla en un portal de noticias aumentó su número de visitas, pero también su audiencia en antena. Posiblemente factores como su oferta generalista y operar en el ámbito local propiciaron esta sinergia (Martínez-Costa; Moreno; Amoedo, 2018).

“En tres meses el portal rac1.cat pasó de 250.000 usuarios únicos mensuales a más de 700.000, según datos de la empresa ComScore. RAC 1 cerró la temporada con los mejores resultados de audiencia de su historia: más de 800.000 oyentes diarios" (Piqué-Abadal, 2018, p. 1).

Ahora incluso en Deportes, la información marca la pauta y denota la diferencia entre los comienzos y la actualidad. Para el periodista J. M. Pou, responsable de las trasmisiones deportivas del Fútbol Club Barcelona, la hibridación de géneros es una característica que define en buena parte el estilo de $R A C 1$. El resultado es una radio "desinhibida"s, en la que los asuntos son abordados desde diferentes perspectivas, una más informativa, otra más opinativa y otra quizá más divertida y cuyo estilo es "informal pero riguroso" (A. Om, entrevista personal, 25 de abril de 2020). Así, el objetivo fue desde el principio distinguirse

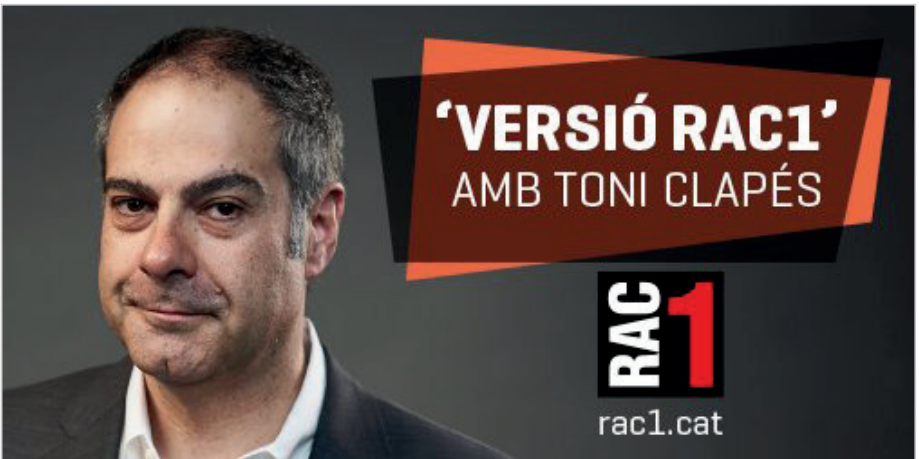

https://api.audioteca.rac1.cat/rsc/img/share/versio.png del resto de marcas radiofónicas.

Como sucede en el conjunto de la radio catalana, en sus inicios, los contenidos deportivos de RAC 1 giraban en torno al Barça. En la actualidad, la variedad es más amplia en cuanto a modalidades deportivas y equipos objeto de interés puesto que como ha ocurrido en otras emisoras/cadenas, el RCD Espanyol cuenta también con un espacio para la retransmisión. Hasta aquí, podría decirse que la diferencia con otras propuestas programáticas es mínima, sin embargo, no es exactamente así ya que emerge la dimensión informativa porque

"hacemos periodismo. Es decir, tenemos complicidad emocional, pero con honestidad. Cuando el Barça lo hace mal, también lo decimos" (J. M. Pou, entrevista personal, 10 de marzo de 2020).

Al igual que el resto de generalistas, el punto de arranque de la jornada radiofónica lo lidera el magazine de prime time, El món a RAC $1^{6}$ y está lógicamente dominado por la actualidad. Según Basté (2020), su director y conductor, las noticias son seleccionadas en base a un factor cronológico.

"Porque cuando la gente se levanta lo que quiere saber es sí ha pasado alguna cosa nueva".

Y se sirven ordenadas sin estigmatización alguna, es decir que

"si la noticia importante es la política va la política, si es económica va la económica, siempre bajo nuestro criterio evidentemente, si deportiva, es deportiva y si es de cine, es de cine .... Hay que buscar una jerarquización que interese a la gente y que tenga ese efecto sorpresivo que les enganche" (J. Basté, entrevista personal, 6 de marzo de 2020).

Debe añadirse que la interacción con la audiencia es alta y no sólo en las redes sociales. Es habitual que, siempre que el director del programa lo considere, entre en antena algún oyente para relatar una problemática personal, aunque se trate del prime time y de la franja horaria más informativa. A partir de esa explicación, el programa se pone en contacto con los actores políticos y/o sociales que pueden dar respuesta a la cuestión planteada. Además, si el tema lo vale, cobra protagonismo en toda la cadena y se realiza un seguimiento del mismo durante días o semanas. Es una diferencia bastante substancial con sus competidoSu audiencia presenta altos índices de fidelización y de identificación con los contenidos que se emiten ras. 


\subsection{El club de la audiencia ${ }^{7}$}

La audiencia forma parte del discurso narrativo de $R A C$ 1, en los contenidos con sus aportaciones, pero también con su participación y fidelidad en los eventos promocionales y sociales que organiza la emisora.

\subsubsection{Creando comunidad}

Según Basté (2020), los oyentes

"se han hecho suya la radio. Se ha creado una especie de secta, donde cabe todo el mundo",

una secta que actualmente es amplia, como evidencia el EGM-Cataluña. La buena sintonía con la emisora es tal que, el hecho de que el catalán sea su lengua vehicular, no supone ningún problema para la audiencia que ni lo habla ni lo escribe, ya que no se trata tanto del vocabulario o de la gramática sino del estilo (J. Basté, entrevista personal, 6 de marzo de 2020). La proximidad con el oyente, el hablarle en un mismo plano es lo que crea esa gran sensación de engagement con la audiencia ${ }^{8}$

"porque hacemos una radio más horizontal que vertical... es una radio líder y transversal... transversal en las edades, es urbana pero rural al mismo tiempo, penetra tan bien en Barcelona como en comarcas. Penetra en el sector catalanohablante pero también en el castellano" (A. Om, entrevista personal, 25 de abril de 2020).

No es un tema de la lengua en la que se emiten los contenidos, para Om es sobre todo por un estilo diferente.

"Una manera de hacer las cosas en la que la gente se siente cómoda ante el micro, independientemente del idioma..., el tipo de programas, el tono y la ideología propician que gente muy diferente, se sienta cómoda en una misma emisora" (A. Om, entrevista personal, 25 de abril de 2020).

Joan-Maria Pou considera que RAC 1 capta oyentes por el contenido de entretenimiento de sus espacios, independientemente de la lengua que use. Se trata de ser empático y de poner proximidad en la comunicación:

“hemos situado el micrófono a pie de calle y hablamos como ellos, porque si hablásemos de forma 'más elevada' nos dirán... no tienes ni puta idea de lo que hablas (Jordi Basté, entrevista personal, 6 de marzo de 2020).

Como Basté, otros presentadores-estrella de RAC 1, ya eran muy reconocidos antes de llegar al Grupo Godó. Ya conocían el medio y tenían larga experiencia radiofónica. Algunos se reinventan en su nueva faceta laboral y otros replican su buen hacer. Por ejemplo, Basté tenía mucha radio deportiva a sus espaldas, primero en Catalunya Ràdio (No ho diguis a ningú), después en RAC 1 (Tu diràs) hasta 2007 que pasa a dirigir y presentar el magazine de prime-time. Pese a cambiar de contenidos, mantiene su tono. Toni Clapés realiza en RAC 1 un magazine de infoentretenimiento, Versió RAC 1 muy parecido en formato, tono y título (con el mismo equipo) al que dirigía anteriormente en Catalunya Ràdio, Versió original. Para ambos, Ilegar a RAC 1 supuso adaptarse a una manera de hacer diferente, quizá más informal y flexible. Para la audiencia catalana eran periodistas conocidos, creíbles y confiables. Este reconocimiento es uno de los factores, señalados anteriormente, como elementos clave del éxito (Martí-Martí, 2000).

\subsubsection{La estrategia digital: una contribución más}

$R A C 1$ nació con la eclosión de internet, un hecho que la hizo

"conceptualmente diferente. Nació en la época de un cambio tecnológico, sin libro de estilo y reconoció que internet es más rápido que la radio" (J. Basté, entrevista personal, 6 de marzo de 2020).

A esta percepción hay que sumarle la juventud de la mayoría de periodistas y realizadores que participaban en el nuevo proyecto radiofónico,

"mayoritariamente de menos de 30 años y que son capaces de crear un tono, un clima que se traduce en un estilo propio y transversal en toda la programación" (Joan-Maria Pou, entrevista personal, 10 de marzo de 2020).

A ello hay que añadir un temprano interés por monitorizar las horas de consumo en streaming, cuando todavía eran pocas las emisoras y cadenas que disponían de dicha información (Ribes, 2008, p. 108). Todos estos factores propiciaron que internet y las redes sociales se integraran de forma natural en la narrativa de los contenidos. De tal manera que, con 675.000 oyentes, el volumen alcanzado de amigos en Facebook (40.252) y seguidores en Twitter (35.020) de El món a $R A C$ 1, el magazín matinal, era significativamente superior a los 19.084 amigos y 14.606 seguidores del programa líder de la radio española, Hoy por hoy (Cadena SER), que contabilizaba 4.285 .000 oyentes (Ribes et al., 2015). Cabe tener en cuenta que, si el índice de audiencia offline permite una mejor negociación con los anunciantes, el fortalecimiento de la marca en internet y redes sociales afianzando "una identidad digital cada vez más reconocible" (Pedrero-Esteban et al., 2017, p. 1101) también beneficia los acuerdos publicitarios.

RAC 1 fue la primera emisora en incorporar aplicaciones para saber, en directo, el comportamiento de los oyentes durante la emisión de un programa. Esta información, traspasada a los realizadores mientras están en antena, puede determinar el rumbo de una entrevista o de un programa. Esta novedad, para los responsables digitales de RAC 1 no es $100 \%$ fiable, pero orienta. 
"Si durante la entrevista ves por internet que la audiencia comienza a bajar significa que aquellos que te escuchan por internet se van. ¿Pero, la gente que está en casa o en el coche, tiene este comportamiento? Esto no lo sabemos, pero es una guía. El locutor puede plantearse si continúa con ese contenido o la abandona (...) Y esto nos permite decidir a qué hora nos conviene hacer circular los contenidos editoriales para las redes sociales.

(Piqué-Abadal, 2018, p. 3)

Otro dato a tener en cuenta es que la producción de contenidos digitales, siguiendo la estrategia de enriquecer la producción de la antena para internet, propicia la consolidación de las atmósferas diferentes que tienen los espacios y sus realizadores. Esta tendencia engrandece la marca corporativa que además se apoya en la reputación de las marcas personales de sus realizadores-estrella.

"Islàndia, el programa de Albert Om a las siete de la tarde, por ejemplo, tiene mucho consumo en las redes sociales. El programa se construye

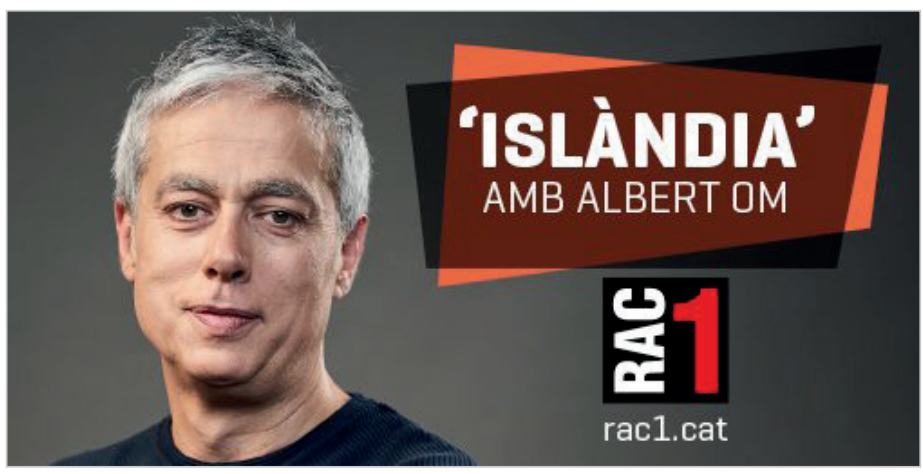

https://api.audioteca.rac1.cat/rsc/img/share/islandia.png a partir de contenidos emocionales, inspiracionales. Apetece dedicar un rato a mirar la web. 'Me conecto, me relajo, escucho el programa, leo la información que hay en la web, envío un mensaje o lo publico en mis redes'. Notamos estos comportamientos” (Piqué-Abadal, 2018 p. 4)

\subsubsection{Público más joven}

Es difícil sin la realización de un estudio de recepción determinar si $R A C 1$ ha tenido y continúa teniendo capacidad para atraer al público más joven de la radio generalista. Para Pou (2020), esta situación es una realidad. De hecho, el podcast con más descargas de Cataluña, según este realizador, es el del programa de humor La competència. Se trata de un espacio que analiza la actualidad desde la ironía más "salvaje" de unos personajes estereotipados, excelentemente guionizados, que son un retrato social divertido. Para uno de los directores, Òscar Dalmau (Blay; Mariné, 2015) esto sólo se puede hacer desde una emisora privada.

"En el fondo, nosotros no dejamos de hacer títeres, un espectáculo de ventriloquia, o sea, que no me imagino alguien levantándose en el teatro y diciéndole al ventrílocuo: “Oye, lo que acaba de decir este personaje...". Bueno, es que este personaje es de broma, es un títere. Por eso te digo que nosotros en este sentido hemos tenido suerte; en el fondo es totalmente normal que la gente que te escucha decodifique que un chiste que te podría parecer hiriente no lo dices como Òscar Dalmau u Òscar Andreu: lo está diciendo un muñeco (Blay; Mariné, 2015)

Para el director del programa de tarde de la emisora, Toni Clapés, La competència, con sus millonarias descargas digitales (entre los mejores podcasts de 2019 en iTunes: RAC1, 2019), y los programas de deportes son los dos espacios que acercaron público joven a la radio.

"Su manera de hablar diferente y poco dogmática... más anárquica, acercó gente joven a la radio. Y después los otros hemos aportado lo que hemos podido" (T. Clapés, entrevista personal, 2020).

Lo que sí es fácilmente rastreable y quizá significativo en este sentido (a pesar de no profundizar en este aspecto en el actual trabajo) es la cantidad de publicidad dirigida a un target universitario y preuniversitario que inunda tanto su web, como sus cortes publicitarios en antena. Sin entrar en más detalles, está claro que La competència y La $2^{\underline{a}}$ hora son nichos publicitarios muy bien segmentados por las agencias que buscan impactar en un target concreto: público joven. De hecho, las conclusiones de un estudio de 2012 para conocer cuál era la programación radiofónica preferida por los estudiantes universitarios catalanes (Pellicer-Muñoz, 2012) puso de manifiesto que después de la radio fórmula musical 40 principales, los universitarios se decantaban por RAC 1, puesto que buscaban contenidos de humor y entretenimiento y una gran parte de su parilla programática se basaba en la actualidad a partir del humor (igual que ahora). Deportes era el otro contenido estimado por los universitarios, aunque en 2012 los podcasts todavía no eran formatos habituales y los jóvenes se quejaban de que este tipo de contenido se programaba cuando estaban en clase. La tendencia actual y las descargas esgrimidas anteriormente refuer-

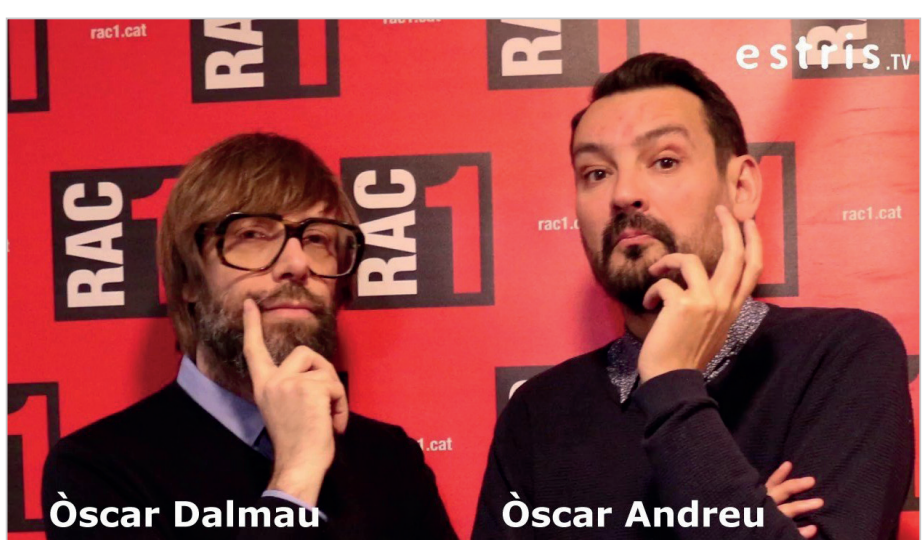
zan esta tesis. 


\subsubsection{Engagement en el marketing}

Hace aproximadamente una década que entró en escena el eslogan de RAC 1 "Tots som 1" (Todos somos uno), una frase que no sólo hace referencia al equipo de profesionales sino también a los oyentes.

"Es un reconocimiento que pone en valor a su audiencia, un activo más de la emisora (A. Om, entrevista personal, 25 de abril de 2020).

La mayoría de los entrevistados considera que este espíritu es un factor que puede explicar también su éxito. Desde el inicio de las emisiones, la privada catalana situó el micrófono en la calle (Basté, 2020), algo que otras también hacían y siguen haciendo. Eso sí, sin la rentabilidad social que le ha reportado a RAC 1.

En términos generales, sus estrategias de promoción y marketing son creativas. Por una parte, cuentan con la complicidad de la audiencia y, por otra con la de los anunciantes que, de esta manera, pueden invertir en campañas muy diferentes a las tradicionales. Así, Turrones Vicens, por ejemplo, patrocinador de varios programas, genera branded content con un concurso en el que los oyentes deciden cada Navidad el tipo de turrón que la marca lanzará con fines solidarios. Sería largo detallar el listado de acciones especiales, comerciales o de autopromoción ${ }^{9}$ que se desarrollan en asociación con la emisora durante una temporada. Naturalmente la publicidad colaborativa o el brand day forma parte del negociado habitual como ocurre en la mayoría de las marcas radiofónicas españolas (Martí-Martí et al., 2015).

Pero, además, RAC 1 ha generado eventos específicos transversales que forman parte de su genuino estilo, contribuyen a su reputación corporativa y son su mejor reclamo publicitario. Son una vuelta modernizada de lo que fueron las grandes campañas de promoción de audiencia de los años 90 (Martí-Martí, 2000). La transversalidad se genera a partir de la colaboración del conjunto de programas y, lógicamente, del papel activo de sus presentadores y periodistas como en el caso, por ejemplo, de És Nadal al món (en referencia al programa El món a RAC 1). Con este evento realizado ante público $^{10}, R A C 1$ felicita las Navidades a los oyentes generando un programa especial, que combina contenidos propios del magazine prime-time y otros nuevos, y que requieren de la presencia activa del resto de conductores de programas. Otra de sus peculiaridades es que sirve de lanzamiento de artistas que acabarán siendo conocidos en el panorama musical. El espectáculo, en un teatro, traspasa la frontera de "sólo radio".

Otra de sus acciones más significativas es la Ràdio cap per avall, una performance que nació del interés de celebrar el Día de la Radio en 2013. Posteriormente, otras emisoras/cadenas se han sumado a la realización de iniciativas en esta línea, pero quizás de una manera más contenida. Tal y como indica el nombre de la programación especial, el 13 de febrero a partir de las 9 de la mañana la oferta es la habitual pero los periodistas actúan en horarios diferentes a los de siempre. Cambio de voces y cambio de carácter para entretener de una forma diferente que ya es un clásico, una marca de la casa. Han conseguido crear expectación ${ }^{11}$.

Ha conseguido modernizar la radio convencional: nacida en la era de internet ha demostrado ser capaz de mejorar y apoyar sus contenidos, monitorizando sus streamings antes que ninguna otra emisora y creando fieles comunidades de amigos

\section{Conclusiones}

Después de comparar las opiniones de los periodistas de RAC 1 con el análisis del contenido de la programación, se observa que la emisora cumple con todos los atributos asociados al éxito, determinados por Martí-Martí (2000; 2008), Gascón-Vera (2016), Guerrero (2013) o Medina (2017). Así, es obvio llegar a la conclusión de que la notoriedad de RAC 1 se fundamenta en la calidad y en sus contenidos disruptivos: ha apostado por la innovación, la creatividad, la originalidad y la experimentación en estructura, en géneros y en la forma de dirigirse y de fidelizar a los oyentes. Ciertamente, sus impulsores conocían el medio y buscaban marcar diferencias con la competencia creando un estilo propio ya desde su arranque hace 20 años. Sin duda, el proveerse de profesionales provenientes básicamente de Catalunya Ràdio favoreció la consolidación de un star system radiofónico muy conocido, entrenado y conocedor del medio, cosa que facilitó una significativa penetración entre la audiencia. En conclusión, los factores que imprimen carácter a la oferta de RAC 1 y la singularizan son:

1) Ruptura de los esquemas clásicos estructurales de la radio generalista que se concretan en:

- Flexibilidad para diluir las franjas horarias.

- Reemisión como estrategia para fortalecer algunos programas y captar oyentes que no pueden escucharlos en sus horarios habituales ni a través de internet.

- Refuerzo del continuum de la emisión beneficiando al contenido por encima de los clásicos parámetros espacio/tiempo de la programación generalista y favoreciendo la fluidez y flexibilidad.

2) Se apostó desde el principio por el infoentretenimiento como género principal y 20 años después continúa siendo el hecho diferencial de la cadena, por encima de la información, cambiando el rol tradicional de los géneros en la radio convencional. El humor, en esta línea, es otro de los emblemas de RAC 1 gracias a programas, llevados a cabo por profesionales pertenecientes a nuevas generaciones que los dotan de contenido muy creativo, sin dejar nada a la improvisación, aunque parezca lo contrario, como es el caso de Minoria absoluta ${ }^{12}$, La competencia o Vostè primer. 
3) Este modelo modernizado de la radio de bloques (Martí-Martí, 2000) ha demostrado tener una alta capacidad para provocar engagement con la audiencia. Los realizadores hablan a los oyentes en un tono muy personalizado y muy cercano. No se trata tanto del vocabulario utilizado o de la gramática del discurso sino de un estilo muy particular. Es la radio transversal que definía Om (2020). Los oyentes participan creando contenidos (prosumers) que la radio resuelve (Basté, 2020). La programación se espectaculariza siempre que es posible, porqué la parrilla en general no es estanca sino muy dinámica y entretenida, y el equipo es joven. Existe una estrategia digital bien diseñada que ayuda al posicionamiento de los programas y de los realizadores como marcas de la corporación, favoreciendo la promoción y la visibilidad de $R A C$ 1. El marketing lanza los eventos especiales de forma llamativa, generando una publicity ${ }^{13}$ que a su vez alimenta más el engagement porque el plan de marketing incorpora tácticas digitales que ayudan en este sentido. Su eslogan "Tots som 1 " es una declaración de principios, que continúa estando vigente.

La penetración de la radio generalista en catalán ha aumentado casi 15 puntos en 10 años. Es altísima, por encima de la radio en español. Desde un share en catalán del 50,6\% hace diez años ${ }^{15}$ al $64 \%$ en 2019, según datos del AIMC-EGM. Esto sucede no porqué la programación sea en catalán, sino porqué, además, tal como se ha expresado en la tesis inicial, la existencia de $R A C 1$ y su nuevo modelo de radio generalista ha conseguido aportar nuevos oyentes y más consumo de un producto entretenido a la par que interesante.

\section{Notas}

1. Este artículo se presentó en formato comunicación al VII Congreso Internacional de la Asociación Española de Investigación de la Comunicación (AE-IC), Valencia, 28-30 de octubre 2020. Por un acuerdo con la $A E-I C$ fue seleccionado, revisado por pares en doble anonimato, y aprobado para ser publicado en esta revista.

2. En esa época, 2011, Manel Fuentes presentaba el talent show “Tu cara me suena” en Antena3.

3. La primera vez que alcanzó el liderazgo en Cataluña fue en febrero-noviembre de 2010 (EGM, 2010, 3a ola), superando a Catalunya Ràdio.

http://reporting.aimc.es/index.htm/\#/main/radio

4. Este trabajo pertenece al Observatori de la Llengua y fue su último informe publicado.

5. Así, definió RAC 1 el entonces President de la Generalitat, Pasqual Maragall, tras ser entrevistado en diciembre del 2000 por Albert Om en el magazine de prime time L'hora del pati.

6. El magazine se estrenó la temporada 2004 dirigido por Xavier Bosch. Después de tres años en la dirección, fue sustituido por Jordi Basté.

7. Ser líder de audiencia ininterrumpidamente en un mercado competitivo como el radiofónico en Cataluña donde incluso la segunda en el ranking, Catalunya Ràdio, emite publicidad a pesar de ser una emisora pública, no es un logro que se vea muy a menudo. Albert Om hacía, en su entrevista para este trabajo, un paralelismo con la SER y RNE en España. La privada se queda con el liderazgo “¿por qué nosotros no podíamos hacer lo mismo aquí?”.

8. El sentido de comunidad ha sido tradicionalmente fuerte entre la audiencia de $R A C 1$, casi con seguridad la primera emisora que contaba con un club de amigos:

http://www.amicsrac1.net

que con motivo del 20 aniversario ha vuelto a activar su banco sonoro y fotográfico.

9. Para ampliar información:

https://aaeegodostrategies.wordpress.com/author/aaeegodostrategies

10. És Nadal al món habitualmente se realiza desde el Palau de la Música Catalana.

11. Para ampliar detalles en este aspecto, ver acciones promocionales de RAC 1:

https://aaeegodostrategies.wordpress.com

12. Desde el inicio de RAC 1 hasta 2009 en antena. Es el embrión del programa Polònia de TV3.

13. Hay que tener presente que La vanguardia del mismo grupo comunicativo de RAC 1, Grupo Godó, contribuye de forma importante a la publicity y a la visibilidad de RAC 1 tanto en noticias como en reportajes.

14. En 2010 las emisoras generalistas con contenido íntegro en catalán eran 4: RAC 1, Catalunya Ràdio, R4 (RNE) i Ona FM. En 2019, el share del EGM se refirió sólo a RAC 1, Catalunya Ràdio y R4, por la desaparición de Ona FM.

\section{Referencias}

Basté, Jordi (2011). Sapigueu que... Barcelona: Columna Edicions. ISBN: 9788466414500

Blay, Blanca; Mariné, Caralp (2015). “Òscar Dalmau: Hem fet conya del conde de Godó i no ha passat res”. El crític, 13 marzo.

https://www.elcritic.cat/entrevistes/oscar-dalmau-hem-fet-conya-del-conde-de-godo-i-no-ha-passat-res-11421 
Butlleti d'informació sobre l'audiovisual a Catalunya (BIAC) (2018), n. 12.

https://mitjansdecomunicacio.gencat.cat/web/.content/Enllac/documents/BIAC11.pdf

Corominas, Maria; Guimerà, Josep-Àngel; Fernández, Isabel; Bonet, Montse (2005). “Polítiques públiques de ràdio i televisió local en l'entorn digital a Espanya (1997-2004)". III Congrés internacional comunicació i realitat. Trípodos, n. extra, pp. 639-650.

https://bit.ly/3uct4Ho

Durán, María-Sol (2015). “Humor negra. Vernaci y el humor en la conducción radiofónica” Letra. Imagen. Sonido (LIS). Ciudad Mediatizada, Año VII, n. 13, pp. 31-38.

https://publicaciones.sociales.uba.ar/index.php/lis/article/view/3788

Espinosa-Mirabet, Sílvia (2013). “Los programas femeninos, una contribución para la historia de la radio”. En: Del-VaIle-Mejías, María-Elena (ed.). La historia y su comunicacion persuaviva. Visión Libros, pp. 79-94. ISBN: 9788415965886

Espinosa-Mirabet, Sílvia; Gutiérrez, Maria; Martí-Martí, Josep-Maria (2019). “Innovative content changes the tendency of consumption in a radio market". En: A. Gazi (moderadora). Talk to me: talk radio cultures. $6^{\text {th }}$ Ecrea Radio Research, 19-21 Sept., University of Siena, Italia.

Fradin, Selma (2016). Les nouveuax business models des médias. Paris: PYP éditions. ISBN: 9782364051362

Gascón-Vera, Patricia (2016). "Claves del éxito de una década televisiva: estudios de caso. El hormiguero y El intermedio". Revista Faro, v. 1, n. 23, pp. 42-71.

https://www.revistafaro.cl/index.php/Faro/article/view/459/440

Guerrero-Pérez, Enrique (2013). Guion y producción de programas de entretenimiento. Navarra: Eunsa. ISBN: 97884 31328962

Gutiérrez, Maria (2008). “L’oferta de programació”. En: Martí-Martí, Josep-Maria; Monclús-Blanco, Belén (eds.). Informe sobre la ràdio a Catalunya, 2006-2007. Observatori de la Ràdio a Catalunya. UAB, pp. 51-68. ISBN: 9788461242917 https://ddd.uab.cat/record/224867

Gutiérrez, Maria (2014) “La programació radiofónica i la consolidació dels models generalista i temàtics”. En: Martí-Martí, Josep-Maria; Monclús-Blanco, Belén (eds.). Informe sobre la ràdio a Catalunya 2007-2011. Observatori de la Ràdio a Catalunya, UAB, pp. 77-99. ISBN: 9788449050947

http://www.l-obsradio.cat/informe-anual-radio.html

Gutiérrez, Maria (2016) “La programació radiofònica: els continguts radiofònics en procés de canvi”. En: Martí-Martí, Josep-Maria; Monclús-Blanco, Belén (eds.). Informe sobre la ràdio a Catalunya, 2012-2014. Observatori de la Ràdio a Catalunya, UAB, pp. 63-81. ISBN: 9788449066436

http://www.l-obsradio.cat/investigacio-detall/informe-sobre-la-radio-a-catalunya-2012-2014/2

Huertas, Amparo; Gutiérrez, Maria (2003). “La programación de las radios generalistas en España”. ZER, n. 8, pp. 117135.

https://ojs.ehu.eus/index.php/Zer/article/view/5365

IAB Spain (2019). Estudio anual de redes sociales

https://iabspain.es/estudio/estudio-anual-de-redes-sociales-2019

Idescat. Estadístiques oficials de Catalunya.

https://www.idescat.cat

La vanguardia (2019) "RAC 1 continúa siendo la radio líder de Catalunya”. La vanguardia, 26 junio.

https://www.lavanguardia.com/vida/20190626/463123644376/rac1-egm-segundo-trimestre-radio-lider-catalunya.html

Llorens-Maluquer, Carles (2019). "La ràdio”. En: Civil-Serra, Marta; López, Bernat (eds.). Informe de la comunicació a Catalunya 2017-2018. Barcelona: Generalitat de Catalunya. Col·lecció Lexikon Informes, n. 6, pp. 161-178.

https://incom.uab.cat/informe/edicion.html?id=17

Martí-Martí, Josep-Maria (2000). De la idea a l'antena. Col.lecció: Eines de periodista. Barcelona: Proa. ISBN: 97884 73066105

Martí-Martí, Josep-Maria (2004). "La oferta radiofónica en Catalunya, entre la madurez y la crisis". Quaderns del CAC, n. 18, pp. 31-36.

Martí-Martí, Josep-Maria (2016). 51 ideas para hacer un buen programa. Barcelona: UOC. ISBN: 9788491164890

Martí-Martí, Josep-Maria; Monclús-Blanco, Belén; Gutiérrez-García, María; Ribes, Xavier (2015). “La radio, modelo de negocio en transición: estrategias de oferta y de comercialización en el contexto digital”. Quaderns del CAC, v. 41, n. 18, pp. 13-22.

https://www.cac.cat/pfw_files/cma/recerca/quaderns_cac/Q41_Marti_et_al_ES.pdf 
Martínez-Costa, María-Pilar; Moreno, Elsa; Amoedo, Avelino (2018). “Mapa de la radio online en España: tipología y caracterización en el contexto de los cibermedios". El profesional de la información, v. 27, n. 4, pp. 849-857.

https://doi.org/10.3145/epi.2018.jul.14

Medina-Ávila, Virginia (2017). "Radio y buen humor. Dos experiencias en la radio latinoamericana: La Luciérnaga en Colombia y El Weso en México. Comunicación y sociedad, n. 28, pp. 155-179.

http://www.scielo.org.mx/pdf/comso/n28/0188-252X-comso-28-00155.pdf

Morros, Joan-Maria (2020). RAC 1: Así se construye un liderazgo.

https://www.lavanguardia.com/vida/20200501/48858086811/rac1-asi-se-construye-un-liderazgo.html

Pedrero-Esteban, Luis-Miguel; Herrera-Damas, Susana (2017). "La notificación push como estrategia informativa de la radio en el entorno digital”. El profesional de la información, v. 26, n. 6, pp. 1100-1107.

https://doi.org/10.3145/epi.2017.nov.09

Pellicer-Muñoz, Àlex (2012). La programació de Catalunya Ràdio i RAC 1 i l'audiència universitària a Catalunya. Trabajo de fin de Grado. Universitat de Girona, UdG.

https://dugi-doc.udg.edu/handle/10256/18398

Piqué-Abadal, Jaume (2018). "L'estratègia digital de RAC 1: De rècord en rècord, i tiro perquè em toca!". Observatori de la Producció Audiovisual (OPA). Dossier Ràdio i xarxes socials.

https://www.upf.edu/documents/8366329/217996117/Miro\%CC\%81AymerichRAC1_editat+\%282+files+merged\%29.pdf

Pradilla-Cardona, Miquel-Àngel; Sorolla-Vidal, Natxo (2016). IX Informe sobre la situació de la llengua catalana: 2015. Barcelona: Observatori de la Llengua Catalana.

http://blogs.iec.cat/cruscat/wp-content/uploads/sites/15/2016/11/informe2015.pdf

RAC (2019). "La 3a Ola del EGM indica un ligero descenso en el consumo de ràdio con un liderazgo consolidado de la radio en catalán". Ràdio Associació de Catalunya. Referència i compromís, 27 noviembre.

http://radioassociacio.cat/la-3a-ola-del-egm-indica-un-ligero-descenso-en-el-consumo-de-radio-con-un-liderazgoconsolidado-de-la-radio-en-catalan

RAC1 (2019). “Apple escull 'La competència' com un dels millors podcasts del 2019”. RAC1, 6 diciembre.

https://www.rac1.cat/cultura/20191203/472036090149/apple-la-competencia-millor-podcasts-itunes-populars-llista.html

Ribes, Xavier (2008). “Informe especial: La ràdio catalana a Internet 2006-2007”. En: Martí-Martí, Josep-Maria; Monclús-Blanco, Belén (eds.). Informe sobre la ràdio a Catalunya, 2006-2007. Observatori de la Ràdio a Catalunya, UAB, pp. 99-113. ISBN: 9788461242917

https://ddd.uab.cat/record/224867

Ribes Xavier; Monclús-Blanco, Belén; Gutiérrez, Maria; Ferrer, Iliana; Martí-Martí, Josep M. (2015). “Crises that Spanish operators face: Marketing and bidding strategies in the digital context". In: K.T. Wilkinson (mod.). Media technology consumption track. Opportunities for media technology consumption. Hispanic/Latino media and marketing international conference. Florida State University, Tallahasee, Florida, USA, 19-21 Febr.

Tresserras, Joan-Manuel (ed.) (2007). Informe sobre l'Audiovisual a Catalunya, CAC, 2005. Barcelona: Consell de l'Audiovisual de Catalunya. ISBN: 9788439373728

Videla-Rodríguez, Juan-José; Sanjuán, Antonio (2006). “La producción independiente de la televisión en España: una radiografía del mercado". En: IX Congreso Ibercom, Sevilla - Cádiz.

https://idus.us.es/handle/11441/34008

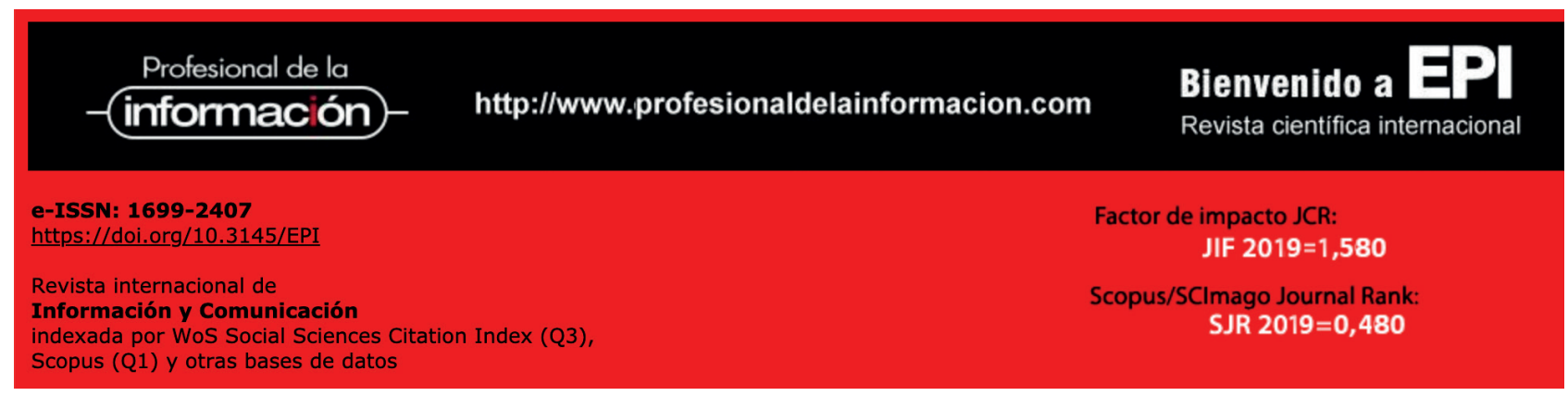

